\section{ACKNOWLEDGMENTS}

The authors wish to thank Dr. J.H. Malmberg and Dr. C. B. Wharton for making their experimental results available prior to publication and for valuable discussions.

\footnotetext{
${ }^{*}$ Research sponsored by the Defense Atomic Support Agency, under Contract No. DASA 01-68-C-0091.

$\dagger$ Present address: Lawrence Radiation Laboratory, Livermore, Calif.

${ }^{1}$ S. J. Buchsbaum and A. Hasegawa, Phys. Rev. 143, A303 (1966).

${ }^{2}$ For a more extensive list of references, see Ref. 1 .

${ }^{3}$ C. B. Wharton and J. H. Malmberg, Proceedings of the Seventh International Conference on Phenomena in Ionized Gases (Gradevinska knjiga, Beograd, Yugoslavia, 1966),
}

\author{
Vol. II, p. 256 . \\ ${ }^{4}$ L. D. Pearlstein and D. Bhadra, Phys. Fluids 12,213 \\ (1969). \\ ${ }^{5}$ J. H. Malmberg, Gulf General Atomic Report No. GA- \\ 8375, Appendix VII (unpublished). \\ ${ }^{6}$ Y. Shima and T. K. Fowler, Phys. Fluids $\underline{8}, 2245$ \\ (1965). \\ ${ }^{7}$ D. K. Bhadra, Gulf General Atomic Report No. \\ GA-8328 (unpublished). \\ ${ }^{8}$ D. K. Bhadra, Phys. Rev. 171, 188 (1968).
}

\title{
Calculation of Current Correlations in Classical Fluids via Modeled Kinetic Equations*
}

\author{
J. J. Duderstadt and A. Z. Akcasu \\ Department of Nuclear Engineering, The University of Michigan, Ann Arbor, Michigan 48105
}

(Received 13 October 1969)

\begin{abstract}
In an earlier paper, the generalized Langevin approach of Mori and Zwanzig has been applied to generate an exact kinetic equation describing the time correlation of fluctuations in the microscopic phase density for a classical many-body system. In the present work, this kinetic equation is modeled using a single-relaxation-time form for the damping kernel, and the solution of this equation is then studied for large and small values of $k$ and $\omega$. The modeled kinetic equation is used to calculate transverse and longitudinal current-current correlations in simple classical liquids, and the results are compared with the molecular-dynamics calculations of Rahman for argonlike systems.
\end{abstract}

\section{INTRODUCTION}

In a recent paper ${ }^{1}$ (hereafter referred to as I), the projection operator techniques of Zwanzig ${ }^{2}$ and Mori $^{3}$ were used to derive an exact kinetic equation for $\mathcal{S}\left(\overrightarrow{\mathrm{x}}, \overrightarrow{\mathrm{p}} ; \overrightarrow{\mathrm{x}}^{\prime}, \overrightarrow{\mathrm{p}}^{\prime}, t\right)$, the time correlation of fluctuations in the microscopic phase density. That is, $\mathcal{G}\left(\overrightarrow{\mathrm{x}}, \overrightarrow{\mathrm{p}} ; \overrightarrow{\mathrm{x}}^{\prime}, \overrightarrow{\mathrm{p}}^{\prime}, t\right)$ represents the probability that, given a particle at $(\overrightarrow{\mathrm{x}}, \overrightarrow{\mathrm{p}})$ at time $t=0$, there will be a particle at $\left(\overrightarrow{\mathrm{x}}^{\prime}, \overrightarrow{\mathrm{p}}^{\prime}\right)$ at a later time $t$. This function plays an important role in the study of many-body systems, since most time correlation functions of interest can be generated by taking suitable moments of $\mathcal{G}$ in momentum $\overrightarrow{\mathrm{p}}$. The purpose of this paper is to use this kinetic-equation description of $\mathcal{G}$ to calculate transverse and longitudinal current-current correlations in simple classical fluids. In particular, these calculations will be compared with the molecular-dynamics calculations of Rahman, as well as with other con- temporary theories of time correlation functions in liquids. ${ }^{5,6}$

To allow a detailed investigation of the solution to the kinetic equation, we will introduce an approximation by modeling the damping or memory term in this equation by a "single-relaxation-time" collision operator in analogy to the work of others. ${ }^{6,7}$ Of course, one could introduce alternative approximations to this term (several alternative approximations were suggested in I). However, the modeling approximation adopted here yields results which are in good agreement with the computer experiments of Rahman, and, in fact, represents a considerable improvement over existing kinetic descriptions of time correlation functions in simple liquids. ${ }^{8,9}$

In this paper, we will also compare the kineticequation description of time correlation functions (i. e., that description based upon the procedure of first solving a kinetic equation for $\mathcal{G}\left(\overrightarrow{\mathrm{x}}, \overrightarrow{\mathrm{p}} ; \overrightarrow{\mathrm{x}}^{\prime}, \overrightarrow{\mathrm{p}}^{\prime}, t\right)$ 
and then taking the appropriate moments in momentum to generate the time correlation function of interest) with the more direct calculation of these quantities via a "generalized hydrodynamic equation" level of description. This latter approach, which has been developed by Martin, Yip, et $a l .{ }^{6,10}$ and also by Akcasu and Daniels, ${ }^{5}$ seeks to obtain hydrodynamiclike equations which directly describe the time correlation functions of interest. Of course, one might expect the kinetic description to yield better results for large $k$ and $\omega$, whereas the hydrodynamic description would be more suitable for small $k$ and $\omega$. However, we will attempt a more thorough comparison of these approaches, and in particular will demonstrate that, under suitable approximations, these descriptions yield similar results over an overlapping region of $k$ and $\omega$.

\section{MODELED KINETIC EQUATION FOR $G\left(\overrightarrow{\mathbf{x}}, \overrightarrow{\mathbf{p}} ; \overrightarrow{\mathbf{x}}^{\prime}, \overrightarrow{\mathbf{p}}^{\prime}, t\right)$}

Consider a vector $\underline{\text { a }}$ whose components $a_{j}$ are dynamical variables of the phase $\left(\overrightarrow{\mathrm{x}}^{1}, \ldots, \overrightarrow{\mathrm{x}}^{N}\right.$, $\overrightarrow{\mathrm{p}}^{1}, \ldots, \overrightarrow{\mathrm{p}}^{N}$ ) of a many-body system. By utilizing projection operators, Mori ${ }^{3}$ has obtained an exact equation for the correlation matrix $\underline{\Gamma}(t)=\left\langle\underline{a}(t) \underline{a}^{*}(0)\right\rangle$ $\times\langle\underline{a}(0) \underline{a} *(0)\rangle^{-1}$ which assumes the form

$$
\frac{d}{d t} \underline{\Gamma}(t)-i \underline{\Omega} \cdot \underline{\Gamma}(t)+\int_{0}^{t} d \tau \underline{\varphi}(\tau) \cdot \underline{\Gamma}(t-\tau)=0 .
$$

In I, this equation was applied to the study of correlations in fluctuations in the microscopic phase density $\delta g(\overrightarrow{\mathrm{x}}, \overrightarrow{\mathrm{p}}, t)$, or equivalently, its Fourier transform in space $\delta g(\overrightarrow{\mathrm{k}}, \overrightarrow{\mathrm{p}}, t)$. That is, a $(t)$ was chosen to be a "vector" whose "components" $a_{j} \rightarrow a(\overrightarrow{\mathrm{p}}, t)$ are indexed by a continuous parameter $\overrightarrow{\mathrm{p}}$ and defined by

$$
\begin{aligned}
& a(\overrightarrow{\mathrm{p}}, t) \equiv \delta g(\overrightarrow{\mathrm{k}}, \overrightarrow{\mathrm{p}}, t) \\
& \quad=\sum_{\alpha=1}^{N} e^{i \overrightarrow{\mathrm{k}} \cdot \overrightarrow{\mathrm{x}}^{\alpha}(t)} \delta\left[\overrightarrow{\mathrm{p}}-\overrightarrow{\mathrm{p}}^{\alpha}(t)\right]-n \delta(\overrightarrow{\mathrm{k}}) M(\overrightarrow{\mathrm{p}}),
\end{aligned}
$$

where it has been noted that $\langle g(\overrightarrow{\mathrm{k}}, \overrightarrow{\mathrm{p}}, t)\rangle=n \delta(\overrightarrow{\mathrm{k}}) M(\overrightarrow{\mathrm{p}})$, $n$ being the equilibrium number density, and

$$
M(\vec{p}) \equiv(\beta / 2 \pi m)^{3 / 2} \exp \left(-\beta p^{2} / 2 m\right)
$$

Mori's equation (1) for the corresponding "correlation matrix"

$$
\oint_{k}\left(\overrightarrow{\mathrm{p}}, \overrightarrow{\mathrm{p}}^{\prime \prime}, t\right) \equiv\left\langle\delta g(\overrightarrow{\mathrm{k}}, \overrightarrow{\mathrm{p}}, t) \delta g^{*}\left(\overrightarrow{\mathrm{k}}, \overrightarrow{\mathrm{p}}^{\prime \prime}, 0\right)\right\rangle
$$

then becomes a linear kinetic equation

$$
\frac{\partial S_{k}}{\partial t}-\frac{i \overrightarrow{\mathrm{k}} \cdot \overrightarrow{\mathrm{p}}}{m} \Theta_{k}\left(\overrightarrow{\mathrm{p}}, \overrightarrow{\mathrm{p}}^{\prime \prime}, t\right)
$$

$$
\begin{aligned}
& +\frac{i \overrightarrow{\mathrm{k}} \cdot \overrightarrow{\mathrm{p}}}{m} M(\overrightarrow{\mathrm{p}}) \frac{n g(k)}{1+n h(k)} \int d \overrightarrow{\mathrm{p}}^{\prime} \mathcal{G}_{k}\left(\overrightarrow{\mathrm{p}}^{\prime}, \overrightarrow{\mathrm{p}}^{\prime \prime}, t\right) \\
& =-\int_{0}^{t} d \tau \int d \overrightarrow{\mathrm{p}}^{\prime} \varphi\left(\overrightarrow{\mathrm{p}}, \overrightarrow{\mathrm{p}}^{\prime}, \tau\right) \mathcal{G}_{k}\left(\overrightarrow{\mathrm{p}}^{\prime}, \overrightarrow{\mathrm{p}}^{\prime \prime}, t-\tau\right) .
\end{aligned}
$$

Here, $g(r)$ is the static pair correlation function, and $h(r)=g(r)-1$. The "damping kernel" $\varphi(\vec{p}, \overrightarrow{\mathrm{p}}, \tau)$ can be calculated ${ }^{1}$ as

$$
\begin{aligned}
& \varphi\left(\overrightarrow{\mathrm{p}}, \overrightarrow{\mathrm{p}}^{\prime}, \tau\right)=[n M(\overrightarrow{\mathrm{p}})]^{-1} \\
& \quad \times\left\langle(1-P) \sigma^{*}\left(\overrightarrow{\mathrm{p}}^{\prime}\right) \exp [i \tau(1-P) L](1-P) \sigma(\overrightarrow{\mathrm{p}})\right\rangle,(5)
\end{aligned}
$$

where we define the dynamical variable $\sigma(\overrightarrow{\mathrm{p}})$ by

$$
\sigma(\overrightarrow{\mathrm{p}}) \equiv \sum_{\alpha=1}^{N} e^{i \overrightarrow{\mathrm{k}} \cdot \overrightarrow{\mathrm{x}}^{\alpha}} \overrightarrow{\mathrm{F}}^{\alpha} \cdot \frac{\partial}{\partial \overrightarrow{\mathrm{p}}^{\alpha}} \delta\left(\overrightarrow{\mathrm{p}}-\overrightarrow{\mathrm{p}}^{\alpha}\right) .
$$

Here, $L$ is the Liouville operator $L=i\{H, \cdot\}$, and $P$ is a projection operator defined by its action on an arbitrary dynamical variable $G(\overrightarrow{\mathrm{p}})$ as

$$
\begin{gathered}
P G(\overrightarrow{\mathrm{p}}) \equiv \int d \overrightarrow{\mathrm{p}} !^{\prime} \int d \overrightarrow{\mathrm{p}}^{\prime \prime}\left\langle G(\overrightarrow{\mathrm{p}}) \delta g^{*}\left(\overrightarrow{\mathrm{k}}, \overrightarrow{\mathrm{p}}^{\prime}, 0\right)\right\rangle \\
\quad \times \phi^{-1}\left(\overrightarrow{\mathrm{p}}^{\prime}, \overrightarrow{\mathrm{p}}^{\prime \prime}\right) \delta g\left(\overrightarrow{\mathrm{k}}, \overrightarrow{\mathrm{p}}^{\prime \prime}, 0\right), \\
\text { with } \quad \phi^{-1}\left(\overrightarrow{\mathrm{p}}^{\prime}, \overrightarrow{\mathrm{p}}^{\prime \prime}\right)=\frac{\delta\left(\overrightarrow{\mathrm{p}}^{\prime}-\overrightarrow{\mathrm{p}}^{\prime \prime}\right)}{n M\left(\overrightarrow{\mathrm{p}}^{\prime}\right)}-\frac{h(k)}{1+n h(k)} .
\end{gathered}
$$

[For details concerning the derivation of Eq. (4), refer to I. ]

Equation (4) is an exact kinetic equation for $S_{k}$. Hence, before we can attempt to solve it, we must first introduce approximations to reduce the damping term to a more tractible form. Of course, the most direct approximation would be to simply neglect the damping term. In this approximation, Eq. (4) reduces to the Vlasov-like equation studied by Zwanzig, ${ }^{11}$ Nelkin and Ranganathan, ${ }^{8}$ and Chihara. ${ }^{12}$ Unfortunately, as noted by these authors, this approximate kinetic equation yields satisfactory results only for very large $k$ and $\omega$, and hence is closely related to a "short time" or "collisionless" approximation.

Alternative approximations of the damping term as suggested in I were based either upon a perturbation calculation of $\varphi\left(\vec{p}, \vec{p}^{\prime}, \tau\right)$ or upon a multicomponent kinetic description closely related to Mori's continued fraction representation of time correlation functions. ${ }^{13}$ Both of these approaches yield integrodifferential kinetic equations which, while very suggestive in appearance, are in fact rather difficult to solve. To circumvent these difficulties, we will adopt a "modeled" description of the damping term as follows: Assume that the time behavior of the damping kernel $\varphi\left(\vec{p}, \vec{p}^{\prime}, \tau\right)$ is exponential ${ }^{14}$ such that 


$$
\varphi\left(\overrightarrow{\mathrm{p}}, \overrightarrow{\mathrm{p}}^{\prime}, \tau\right)=\varphi\left(\overrightarrow{\mathrm{p}}, \overrightarrow{\mathrm{p}}^{\prime}, 0\right) \exp [-\alpha(k) \tau],
$$

where the exact form for $\varphi\left(\vec{p}, \vec{p}^{\prime}, 0\right)$ will be used, while $\alpha(k)$ will be determined at a later stage of the calculation. This "single-relaxation-time" approximation is very similar to that introduced earlier by Berne, Boon, and Rice ${ }^{7}$ in the calculation of the momentum autocorrelation function of a test particle in a liquid - except that we allow a $k$-dependent relaxation constant $\alpha(k)$. Such an approximation can be motivated by considering a two-component kinetic description (see I) and recognizing that one expects the damping kernel to be a rapidly decaying function of time [at least on the time scale of $g_{k}(t)$ ]. However, we will regard (9) as merely a model and, as such, justified only by agreement with experimental data.

It is a straightforward task to evaluate $\varphi(\overrightarrow{\mathrm{p}}, \overrightarrow{\mathrm{p}}, 0)$ from equation (5) (see Appendix A for details). Such a calculation yields

$$
\begin{aligned}
\int d \overrightarrow{\mathrm{p}}^{\prime} \varphi\left(\overrightarrow{\mathrm{p}}, \overrightarrow{\mathrm{p}}^{\prime}, 0\right) \Theta_{k}\left(\overrightarrow{\mathrm{p}}^{\prime}, \overrightarrow{\mathrm{p}}^{\prime \prime}, t\right) \\
=-D(0)\left\{\frac{\partial}{\partial \overrightarrow{\mathrm{p}}} \cdot \frac{\partial}{\partial \overrightarrow{\mathrm{p}}}+\frac{\beta}{m} \frac{\partial}{\partial \overrightarrow{\mathrm{p}}} \cdot \overrightarrow{\mathrm{p}}\right\} \oint_{k}\left(\overrightarrow{\mathrm{p}}, \overrightarrow{\mathrm{p}}^{\prime \prime}, t\right) \\
+\overrightarrow{\mathrm{p}} M(\overrightarrow{\mathrm{p}}) \cdot \vec{D}(k) \cdot \int d \overrightarrow{\mathrm{p}}^{\prime} \overrightarrow{\mathrm{p}}^{\prime} \varrho_{k}\left(\overrightarrow{\mathrm{p}}^{\prime}, \overrightarrow{\mathrm{p}}^{\prime \prime}, t\right),
\end{aligned}
$$

where $\vec{D}(k) \equiv \vec{k} \vec{k} n c(k) / m^{2}-\beta^{2} / m^{2} \overrightarrow{\mathrm{D}}(k)$,

$$
\begin{aligned}
D_{i j}(k) & \equiv(n / \beta) \int d \overrightarrow{\mathrm{x}} \frac{\partial^{2} V}{\partial x_{j}^{2}} g(x) \cos k x_{3} \delta_{i j}, \\
D(0) & \equiv(n / \beta) \int d \overrightarrow{\mathrm{x}} \frac{\partial^{2} V}{\partial x_{j}^{2}} g(x),
\end{aligned}
$$

and $c(k)=g(k) /[1+n h(k)]$ is the direct correlation function, while $V(r)$ is the interparticle potential. Hence, the modeled kinetic equation we will consider becomes, after a Laplace transform in time,

$$
\begin{aligned}
& \left(s-\frac{i \overrightarrow{\mathrm{k}} \cdot \overrightarrow{\mathrm{p}}}{m}\right) \tilde{\Theta}_{k}\left(\overrightarrow{\mathrm{p}}, \overrightarrow{\mathrm{p}}^{\prime \prime}, s\right)+\frac{i \overrightarrow{\mathrm{k}} \cdot \overrightarrow{\mathrm{p}}}{m} M(\overrightarrow{\mathrm{p}}) n c(k) \\
& \quad \times \int d \overrightarrow{\mathrm{p}}^{\prime} \tilde{\Theta}_{k}\left(\overrightarrow{\mathrm{p}}^{\prime}, \overrightarrow{\mathrm{p}}^{\prime \prime}, s\right)=\Theta_{k}\left(\overrightarrow{\mathrm{p}}, \overrightarrow{\mathrm{p}}^{\prime \prime}, 0\right) \\
& \quad+\frac{D(0)}{s+\alpha(k)}\left\{\frac{\partial}{\partial \overrightarrow{\mathrm{p}}} \cdot \frac{\partial}{\partial \overrightarrow{\mathrm{p}}}+\frac{\beta}{m} \frac{\partial}{\partial \overrightarrow{\mathrm{p}}} \cdot \overrightarrow{\mathrm{p}}\right\} \mathrm{S}_{k}\left(p, p^{\prime \prime}, s\right) \\
& \quad-\frac{\overrightarrow{\mathrm{p}} M(\overrightarrow{\mathrm{p}}) \cdot \vec{D}(k)}{s+\alpha(k)} \cdot \int d \overrightarrow{\mathrm{p}}^{\prime} \overrightarrow{\mathrm{p}}^{\prime} \tilde{\mathrm{S}}_{k}\left(\overrightarrow{\mathrm{p}}^{\prime}, \overrightarrow{\mathrm{p}}^{\prime \prime}, s\right),
\end{aligned}
$$

where $\mathcal{G}_{k}\left(\overrightarrow{\mathrm{p}}, \overrightarrow{\mathrm{p}}^{\prime \prime}, 0\right)=n M(\overrightarrow{\mathrm{p}}) \delta\left(\overrightarrow{\mathrm{p}}-\overrightarrow{\mathrm{p}}^{\prime \prime}\right)$

$$
+n^{2} M(\overrightarrow{\mathrm{p}}) M\left(\overrightarrow{\mathrm{p}}^{\prime \prime}\right) h(k) \text {. }
$$

\section{CALCULATION OF TRANSVERSE} CURRENT CORRELATIONS

If we define the microscopic current density $\vec{J}(\vec{k}, 0)$ by

$$
\overrightarrow{\mathrm{J}}(\overrightarrow{\mathrm{k}}, 0) \equiv \sum_{\alpha=1}^{N}\left(\overrightarrow{\mathrm{p}}^{\alpha} / m\right) \exp \left(i \overrightarrow{\mathrm{k}} \cdot \overrightarrow{\mathrm{x}}^{\alpha}\right),
$$

then the transverse current-current correlation function

$$
\mathfrak{J}_{\perp}(\overrightarrow{\mathrm{k}}, t) \equiv\left\langle J_{1}^{*}(\overrightarrow{\mathrm{k}}, 0) J_{1}(\overrightarrow{\mathrm{k}}, t)\right\rangle\left\langle J_{1}^{*}(\overrightarrow{\mathrm{k}}, 0) J_{1}(\overrightarrow{\mathrm{k}}, 0)\right\rangle^{-1}
$$

can be written in terms of $\mathcal{S}_{k}\left(\overrightarrow{\mathrm{p}}, \overrightarrow{\mathrm{p}}^{\prime \prime}, t\right)$ as

$$
\mathcal{g}_{\perp}(\overrightarrow{\mathrm{k}}, t)=(\beta / m n) \int d \overrightarrow{\mathrm{p}} p_{1} \int d \overrightarrow{\mathrm{p}}^{\prime \prime} p_{1}^{\prime \prime} \mathrm{g}_{k}\left(\overrightarrow{\mathrm{p}}, \overrightarrow{\mathrm{p}}^{\prime \prime}, t\right),
$$

where we choose our coordinate system $\left(p_{1}, p_{2}, p_{3}\right)$ such that $p_{3}$ is parallel to $\overrightarrow{\mathrm{k}}$. Hence, to calculate $g_{\perp}(\vec{k}, t)$, we need only compute a moment of $\mathcal{S}_{k}$.

Rather than attempting to solve the modeled kinetic equation (12) for $\oint_{k}\left(\overrightarrow{\mathrm{p}}, \overrightarrow{\mathrm{p}}^{\prime \prime}, s\right)$ directly, we will instead first multiply it by $p_{1}$ and $p_{1}^{\prime \prime}$ and then integrate over $\overrightarrow{\mathrm{p}}^{\prime \prime}$ and $p_{1}$ and $p_{2}$ to find

$$
\begin{gathered}
\left(s-\frac{i k p}{m}\right) X(p, s)-\frac{D(0)}{s+\alpha(k)}\left[\frac{d^{2}}{d p^{2}}+\frac{\beta}{m} p \frac{d}{d p}\right] X(p, s) \\
-\frac{(\beta / m) D_{11}(k) M(p)}{s+\alpha(k)} \int_{-\infty}^{+\infty} d p^{\prime} X\left(p^{\prime}, s\right) \\
=(m n / \beta) M(p)
\end{gathered}
$$

where we have denoted $p_{3} \rightarrow p$ and defined

$$
X(p, s) \equiv \int_{-\infty}^{\infty} d p_{1} \int_{-\infty}^{\infty} d p_{2} p_{1} \int d \overrightarrow{\mathrm{p}}^{\prime \prime} p_{1}^{\prime \prime} \tilde{\Theta}_{k}\left(\overrightarrow{\mathrm{p}}, \overrightarrow{\mathrm{p}}^{\prime \prime}, s\right) .
$$

Equation (17) is now an integrodifferential equation in the single variable $p$.

Recognize that we need only compute the integral of $X(p, s)$ over all $p$ to arrive at $\tilde{J}_{\perp}(k, s)$. With this in mind, let us study Eq. (17) in two limiting cases: In the limit of large $s$ it is evident that we can neglect the damping term in (17) to find

$$
\tilde{J}_{\perp}(k, s)=\left(\frac{m \beta}{2 \pi}\right)^{1 / 2} \int_{-\infty}^{\infty} d p \frac{e^{-p^{2} \beta / 2 m}}{s-i k p / m} .
$$

Of more interest is the cosine transform of $\partial_{\perp}(k, t)$ in time given by

$$
d_{\perp}(k, \omega)=\operatorname{Re}\left\{\lim _{\epsilon \rightarrow 0^{+}} \tilde{J}_{\perp}(k, i \omega+\epsilon)\right\}
$$




$$
=\left(\frac{\pi m \beta}{2 k^{2}}\right)^{1 / 2} \exp \left(-\frac{\omega^{2}}{k^{2}}\left(\frac{1}{2} m \beta\right)\right) .
$$

Of course, this is just the form of the transverse current correlation one would have obtained for an ideal gas - that is, a system in which there are no particle interactions. This is the same result which one obtains from the Vlasov kinetic equation for a liquid, ${ }^{8}$ hence verifying that the total neglect of the damping term is related to a large $\omega$ or short-time approximation.

Having verified that our modeled kinetic equation yields the correct large $\omega$ behavior, let us now examine the other extreme of small $k$ and $\omega$. The most convenient approach is to multiply Eq. (17) by $p^{r}$ and integrate over $p$, hence obtaining a recursion relation among the moments $X^{(r)}(s)$ :

$$
\begin{aligned}
& s X^{(r)}-(i k / m) X^{(r+1)}-\frac{D(0)}{s+\alpha(k)} \\
& \times\left(r(r-1) X^{(r-2)}-\frac{\beta}{m}(r+1) X^{(r)}\right)=0, \quad r \text { odd }(21) \\
& s X^{(r)}-(i k / m) X^{(r+1)}-\frac{D(0)}{s+\alpha(k)} \\
& \quad \times\left(r(r-1) X^{(r-2)}-\frac{\beta}{m}(r+1) X^{(r)}\right) \\
& \quad-\frac{D_{11}(k)}{s+\alpha(k)}\left(\frac{m}{\beta}\right)^{r / 2-1}[1 \times 3 \times \cdots \times(r-1)] X^{(0)} \\
& =\left(\frac{m}{\beta}\right)^{r / 2+1} n[1 \times 3 \times \cdots \times(r-1)], \quad r \text { even }
\end{aligned}
$$

where $\quad X^{(r)}(s) \equiv \int_{-\infty}^{\infty} d p p^{r} X(p, s)$.

In particular, note that

$$
\tilde{g}_{\perp}(k, s)=(\beta / m n) X^{(0)}(s) .
$$

It is a straightforward task to compute the first few moments to find

$$
\begin{aligned}
X^{(0)}(s)= & \left(s+\frac{\beta / m\left[D(0)-D_{11}(k)\right]}{s+\alpha(k)}\right)^{-1} \\
& \times\left(\frac{m n}{\beta}+\frac{i k}{m} X^{(1)}\right), \\
X^{(1)}(s)= & \left(s+\frac{2 \beta / m D(0)}{s+\alpha(k)}\right)^{-1}\left(\frac{i k}{m} X^{(2)}\right), \\
X^{(2)}(s)= & \left(s+\frac{3 \beta / m D(0)}{s+\alpha(k)}\right)^{-1}
\end{aligned}
$$

$$
\times\left[\left(\frac{m}{\beta}\right)^{2} n+\left(\frac{2 D(0)+D_{11}(k)}{s+\alpha(k)}\right) X^{(0)}+\frac{i k}{m} X^{(3)}\right] .
$$

- Now note

$$
\frac{\beta}{m}\left[D(0)-D_{11}(k)\right]=\frac{k^{2} C_{44}(k)}{m n}\left(1-\frac{n}{\beta C_{44}(k)}\right),
$$

where $C_{44}(k)$ has been identified as one of the elastic moduli ${ }^{15}$

$$
C_{44}(k)=\frac{n}{\beta}+n^{2} \int d^{3} R g(R) \frac{\partial^{2} V}{\partial x_{1}^{2}}\left(\frac{1-\cos k x_{3}}{k^{2}}\right) .
$$

Hence, from (24) and (25) we find

$$
\begin{aligned}
\mathfrak{J}_{\perp}(k, s)= & \left\{s+\frac{k^{2} C_{44}(k)}{m n} \frac{\left[1-n / \beta C_{44}(k)\right]}{s+\alpha(k)}\right\}^{-1} \\
& \times[1+\delta(k, s)],
\end{aligned}
$$

where we have defined

$$
\delta(k, s) \equiv(\beta / m n)(i k / m) X^{(1)}(s) .
$$

Interestingly enough, this form is very similar to the exact expression derived via a generalized hydrodynamic approach by Akcasu and Daniels ${ }^{3}$ :

$$
\tilde{J}_{\perp}(k, s)=\left\{s+\frac{k^{2} C_{44}(k)}{m n} \frac{1}{s+\tilde{\varphi}_{22}(s)}\right\}^{-1} .
$$

(For more details on this result, refer to Appendix B.) We can now determine $\alpha(k)$ by comparing (30) and the exact hydrodynamic result (32) at $s=0$ to find

$$
\alpha(k)=\omega_{\perp}(k)\left(\frac{1-n / \beta C_{44}(k)}{1+\delta(k, s)}\right) .
$$

Since $\omega_{\perp}(k)=C_{44}(k) / \eta_{\perp}(k, 0)$ (see Appendix B), we find that $\alpha(k)$ is related to a transport parameter, the $k$-dependent shear viscosity. Furthermore, using the relation $\omega_{\perp}(k)=\tilde{\varphi}_{22}(k, 0)$, we can obtain an expression for $\alpha(k)$ in terms of the projection operator introduced in the hydrodynamic description.

The resemblance of the form of (30) to (32) is very suggestive. Indeed, since the additional term $\delta(k, s)$ in $(30)$ is due to the streaming of the particles, represented by $(i k p / m) X(p, s)$ in (17), we may therefore argue that for dense fluids and small $k$, this term will be small compared to unity. Furthermore, the factor $\left[1-n / \beta C_{44}(k)\right]$ in the denominator of (30) is very close to unity for small values of $k$. Hence for small $k$, the expressions (30) and (32) are equivalent, with $\alpha(k)$ also ap- 
proaching $\omega_{\perp}(k)$ as seen from (33). Thus, the modeled kinetic description (12) yields the correct "hydrodynamic" results for small $k$ with the choice of $\alpha(k)$ given in (33). Of course we still do not know $\omega_{\perp}(k)$. However Akcasu and Daniels have modeled this function by using its known behavior for small and large $k$ (see Appendix B). If we use this modeled form for $\omega_{\perp}(k)$ to compute $\alpha(k)$, we can then calculate $\mathcal{J}_{\perp}(k, \omega)$ explicitly.

This calculation has been performed for argon at $T=76^{\circ} \mathrm{K}, m n=1.407 \mathrm{~g} / \mathrm{cm}^{3}$ using the modeled hydrodynamic result (32) as well as the kinetic description retaining the first three moments and taking $\omega_{\perp}(k)$ as given by (B16). These calculations are compared to the molecular-dynamics data of Rahman ${ }^{4}$ in Fig. 1. Notice that the agreement with Rahman's calculations is rather good in all cases. As one expects, the higher moments in the kinetic description are essentially negligible for $k<1.5 \AA^{-1}$, and the hydrodynamic description yields satisfactory results. However for larger $k$, the kinetic description yields somewhat better results due to its more detailed treatment of the frequency dependence. Figure 2 gives a comparison of the frequency $\omega_{m} \perp(k)$ for which $J_{\perp}(k, \omega)$ assumes a maximum for a given value of $k$ for the various theories, while Fig. 3 plots the magnitude of this maximum.

\section{CALCULATION OF LONGITUDINAL CURRENT-CURRENT CORRELATIONS}

We now turn our attention to the calculation of the longitudinal current-current correlation function

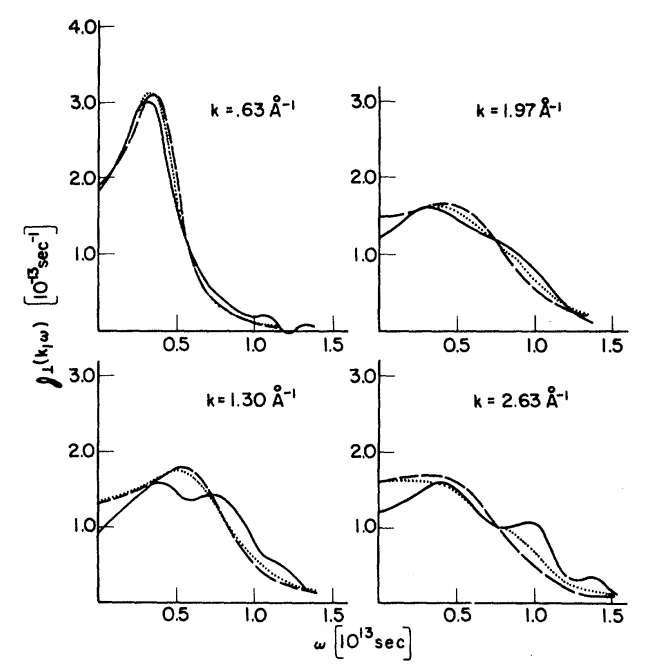

FIG. 1. Transverse current-current correlation function $\mathcal{J}_{\perp}(k, \omega)$ versus $\omega$ and $k$ for argon. The solid curves represent the computer data of Rahman (Ref. 4), the dashed curves represent a single-moment calculation of this work, while the dotted curves represent a threemoment kinetic description.

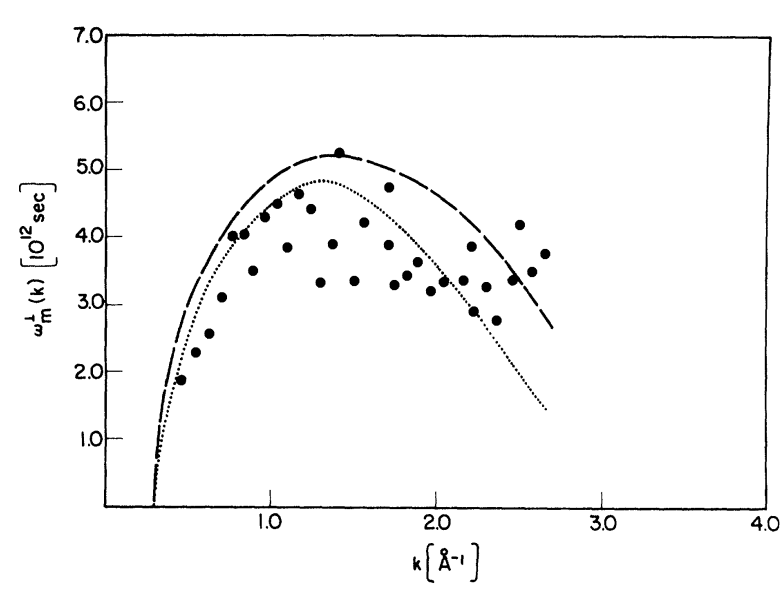

FIG. 2. Frequency $\omega_{m}^{\perp}(k)$ at which $\mathscr{J}_{\perp}(k, \omega)$ assumes a maximum for a given $k$. The points are the data of Rahman, while the dashed and dotted curves are the results of one- and three-moment calculations, respectively.

$$
\mathcal{J}_{\|}(k, t) \equiv\left\langle J_{3}^{*}(\overrightarrow{\mathrm{k}}, 0) J_{3}(\overrightarrow{\mathrm{k}}, t)\right\rangle\left\langle J_{3}^{*}(\overrightarrow{\mathrm{k}}, 0) J_{3}(\overrightarrow{\mathrm{k}}, 0)\right\rangle^{-1}
$$

Recognize that $\mathfrak{d}_{\|}(k, \omega)$ is simply related to the cosine transform, $S(k, \omega)$ of the density-density correlation function

$$
\begin{aligned}
G(k, t) & =\frac{1}{n}\left\langle\rho_{-k}(0) \rho_{k}(t)\right\rangle \\
& =\frac{1}{n} \int d \overrightarrow{\mathrm{p}} \int d \overrightarrow{\mathrm{p}}^{\prime \prime} \mathrm{G}_{k}\left(\overrightarrow{\mathrm{p}}, \overrightarrow{\mathrm{p}}^{\prime \prime}, t\right),
\end{aligned}
$$

by $\mathcal{J}_{\|}(k, \omega)=\left(m \beta \omega^{2} / 2 k^{2}\right) S(k, \omega)$.

We shall apply Eq. (12) to the calculation of $S(k, \omega)$ and hence, obtain $\mathfrak{d}_{\|}(k, \omega)$ from (36). Following the procedure of Sec. III, we first integrate (12) over $\overrightarrow{\mathrm{p}}^{\prime \prime}, p_{1}$, and $p_{2}$ to find

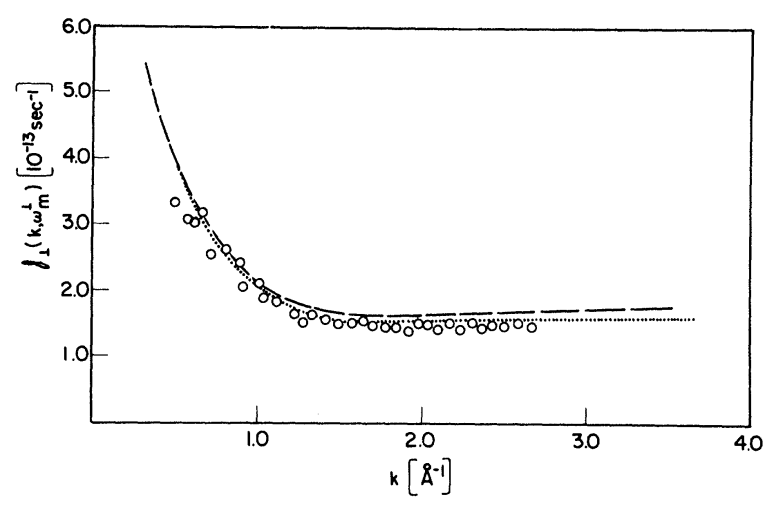

FIG. 3. Value of $\mathcal{J}_{\perp}(k, \omega)$ at $\omega=\omega_{m}^{\perp}(k)$ for various $k$. 


$$
\begin{gathered}
(s-i k p / m) Y(p, s)+\frac{i k p}{m} n c(k) M(p) \int_{-\infty}^{\infty} d p^{\prime} Y\left(p^{\prime}, s\right) \\
-\frac{D(0)}{s+\alpha(k)}\left(\frac{d^{2}}{d p^{2}}+\frac{\beta}{m} \frac{d}{d p} p\right) Y(p, s) \\
+\frac{p M(p)}{s+\alpha(k)}\left(\frac{k^{2}}{m^{2}} n c(k)-\frac{\beta^{2}}{m^{2}} D_{33}(k)\right) \\
\times \int_{-\infty}^{\infty} d p^{\prime} p^{\prime} Y\left(p^{\prime}, s\right)=n M(p) S(k),
\end{gathered}
$$

where again $p_{3} \rightarrow p$ and

$$
Y(p, s) \equiv \int_{-\infty}^{\infty} d p_{1} \int_{-\infty}^{\infty} d p_{2} \int d \overrightarrow{\mathrm{p}}^{\prime \prime} \tilde{\Theta}_{k}\left(\overrightarrow{\mathrm{p}}, \overrightarrow{\mathrm{p}}^{\prime \prime}, s\right) .
$$

Furthermore, $M(p) \equiv(\beta / 2 \pi m)^{1 / 2} \exp \left(-\beta p^{2} / 2 m\right)$. Noting that

$$
S(k, \omega)=\frac{1}{n} \operatorname{Re}\left\{\lim _{\epsilon \rightarrow 0^{+}} \int_{-\infty}^{\infty} d p Y(p, i \omega+\epsilon)\right\},
$$

we again find the large $\omega$ behavior of $S(k, \omega)$ as the solution to (37) without the damping terms

$$
\begin{array}{r}
S(k, \omega)=\left(\frac{\pi m \beta}{2 k^{2}}\right)^{1 / 2} e^{-z^{2}}\left\{\left(\frac{1}{S(k)}+n c(k) 2 z e^{-z^{2}}\right.\right. \\
\left.\left.\times \int_{0}^{z} e^{t^{2}} d t\right)^{2}+\left[n c(k) \sqrt{\pi} z e^{-z^{2}}\right]^{2}\right\}^{-1}, \\
z=\frac{\omega}{k}\left(\frac{1}{2} m \beta\right)^{1 / 2}
\end{array}
$$

This result, first obtained by Nelkin and Ranganathan, ${ }^{8}$ is something of an improvement over the ideal gas result

$$
S(k, \omega)=\left(\frac{\pi}{2} \frac{m \beta}{k^{2}}\right)^{1 / 2} \exp \left(-z^{2}\right),
$$

since it does account for initial correlations. However, it is only valid in a liquid for large $\omega$. In order to study $S(k, \omega)$ over a wider range of $k$ and $\omega$, extending to the values of $k$ and $\omega$ encountered in neutron-scattering experiments, we retain the modeled damping term in (37), multiplying by $p^{r}$ and integrating to obtain a recursion relation among the moments $Y^{(r)}(s)$ :

$$
\begin{array}{cl}
s Y^{(r)}-\frac{i k}{m} Y^{(r+1)}-\frac{D(0)}{s+\alpha(k)}\left[r(r-1) Y^{(r-2)}-\frac{\beta}{m} r Y^{(r)}\right]=n S(k)\left(\frac{m}{\beta}\right)^{r / 2}\left(1 ; 2 ; \frac{1}{2} r\right), & r \text { even, } \\
s Y^{(r)}-\frac{i k}{m} Y^{(r+1)}+\frac{i k}{m}\left(\frac{m}{\beta}\right)(r+1) / 2 n c(k) Y^{(0)}-\frac{D(0)}{s+\alpha^{\prime}(k)}\left(r(r-1) Y^{(r-2)}-\frac{\beta}{m} r Y^{(r)}\right) & \\
+\frac{(m / \beta)}{s+\alpha(k)}\left(1 ; 2 ; \frac{1}{2}(r+1)\right)\left(\frac{k^{2}}{m^{2}} n c(k)-\frac{\beta^{2}}{m^{2}} D_{33}(k)\right) Y^{(1)}=0, & r \text { odd, }
\end{array}
$$

where $(m ; d ; \nu)=m(m+d)(m+2 d) \cdots[m+d(\nu-1)]$ for $\nu=1,2, \ldots$, and $(m ; d ; 0)=1$. More explicitly, the first few moment relations are

$$
\begin{aligned}
s Y^{(0)} & =(i k / m) Y^{(1)}+n S(k), \\
Y^{(1)} & =\left(s+\frac{(\beta / m)\left[D(0)-D_{33}(k)\right]+\left(k^{2} / m \beta\right) n c(k)}{s+\alpha(k)}\right)^{-1}\left(\frac{i k}{m} Y^{(2)}-\frac{i k}{m} n c(k) Y^{(0)}\right), \\
Y^{(2)} & =\left(s+\frac{(2 \beta / m) D(0)}{s+\alpha(k)}\right)^{-1}\left(\frac{n m}{\beta} S(k)+\frac{2 D(0)}{s+\alpha(k)} Y^{(0)}+\frac{i k}{m} Y^{(3)}\right) .
\end{aligned}
$$

One obtains a closed set of equations for the first $(n+1)$ moments by ignoring $Y^{(n+1)}$ in the expression for $Y^{(n)}$. We obtain an approximate expression for $Y^{(0)}$ from the above set by ignoring $Y^{(3)}$ :

$$
Y^{(0)}(s)=\frac{n S(k) Q(k, s)}{s Q(k, s)+k^{2} c_{L}{ }^{2}(k)},
$$

$$
\text { where } \begin{aligned}
Q(k, s) \equiv & s+\frac{k^{2}}{m n} \frac{C_{11}(k)-m n c_{L}{ }^{2}(k)-2 n / \beta}{s+\alpha(k)} \\
& -\frac{k^{2}}{m \beta}\left(s+\frac{2 \beta}{m} \frac{D(0)}{s+\alpha(k)}\right)^{-1} \cdot
\end{aligned}
$$

In obtaining (47) and (48), we have observed that 


$$
\begin{aligned}
\frac{\beta}{m}\left[D(0)-D_{33}(k)\right] & =\frac{k^{2}}{m n} C_{11}(k)-\frac{3 k^{2}}{m \beta}, \\
\frac{k^{2} n c(k)}{m \beta} & =k^{2}\left(\frac{1}{m \beta}-c_{L}^{2}(k)\right),
\end{aligned}
$$

where $C_{11}(k)$ is a component of the elastic modulus tensor, ${ }^{15}$ given by

$$
C_{11}(k)=\frac{3 n}{\beta}+n^{2} \int d^{3} R g(R) \frac{\partial^{2} V}{\partial X_{3}^{2}} \frac{\left(1-\cos k X_{3}\right)}{k^{2}}
$$

in a coordinate system in which $\overrightarrow{\mathrm{k}} \|\left(x_{3}\right.$ axis $)$. The term $c_{L}(k)$ in (48) and (50) is the isothermal speed of sound ${ }^{15}$ defined by

$$
c_{L}^{2}(k) \equiv 1 / m \beta S(k)
$$

where $S(k)$ is the structure factor, i.e., $S(k)=1$ $+n h(k)$. Substituting (47) into (39) and using (36), we obtain the longitudinal current-current correlation function as

$$
\begin{aligned}
J_{11}(k, \omega)= & \operatorname{Re}\left\{i\left(\omega-\frac{k^{2} c_{L}{ }^{2}(k)}{\omega}\right)\right. \\
& \cdot \\
& +\frac{k^{2}}{m n} \frac{C_{11}(k)-m n c_{L}{ }^{2}(k)-2 n / \beta}{i \omega+\alpha(k)}
\end{aligned}
$$

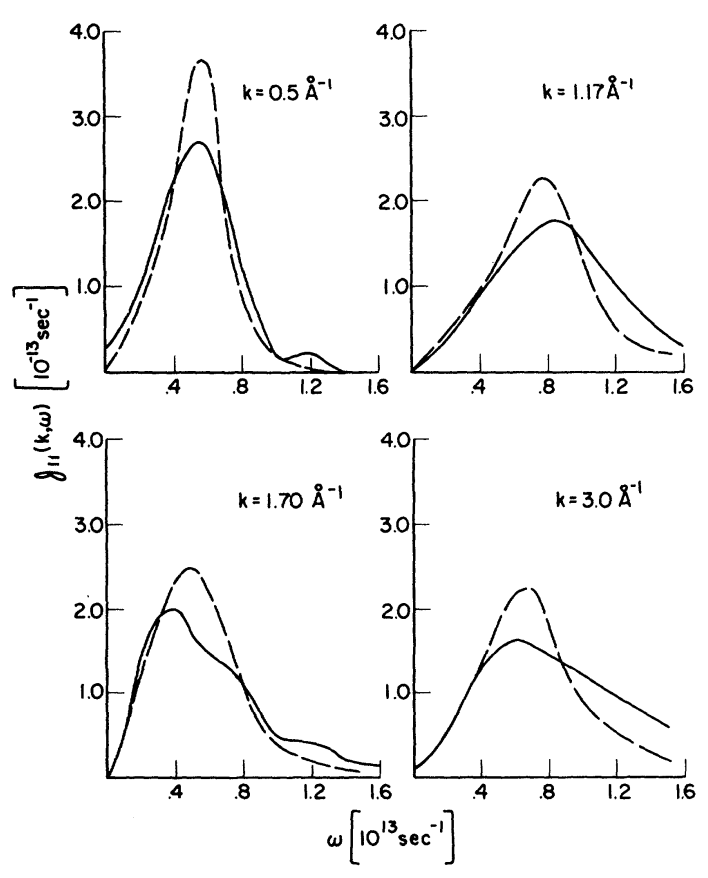

FIG. 4. Longitudinal current-current correlation function $\mathscr{J}_{\|}(k, \omega)$. Again the solid curves are due to Rahman (Ref. 4), whereas the dashed curves correspond to the kinetic-equation description.

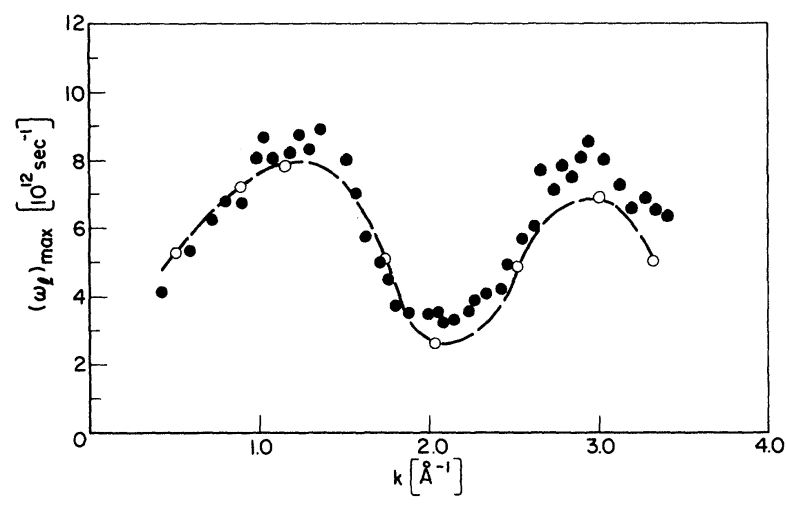

FIG. 5. Frequency $\omega_{m} \|(k)$ at which $\mathscr{J}_{\|}(k, \omega)$ assumes a maximum for a given $k$.

$$
\left.-\frac{k^{2}}{m \beta}\left(i \omega+\frac{2 \beta}{m} \frac{D(0)}{i \omega+\alpha(k)}\right)^{-1}\right\}^{-1}
$$

which again corresponds to the form obtained via the generalized hydrodynamic description. ${ }^{5,6}$

Using the expression (53) we have computed the longitudinal current-current correlation function for argon at $T=76^{\circ} \mathrm{K}, \rho_{0}=m n=1.407 \mathrm{~g} / \mathrm{cm}^{3}$ and compared the results with Rahman ${ }^{4}$ in Figs. 4 and 5. The quantities $c_{L}(k), C_{11}(k)$, and $D(0)$ were computed from (52), (51), and (11), respectively. The relaxation frequency $\alpha(k)$ was taken to be the same as that used in Sec. III when computing the transverse current correlation. [It might be mentioned at this point that the kinetic approach we have adopted here differs from the hydrodynamic approach ${ }^{5}$ in that only one relaxation parameter $\alpha(k)$ is sufficient to yield both transverse and longitudinal current-current correlations. In the hydrodynamic description, ${ }^{5}$ two such relaxation parameters $\omega_{\perp}(k)$ and $\omega_{\|}(k)$ were necessary. ] Again the comparison of the moment calculations with Rahman's data seems to be quite reasonable.

\section{CONCLUSIONS}

By adopting a single-relaxation-time model of the damping kernel in the exact kinetic equation developed in $\mathrm{I}$, we have been able to explicitly calculate current-current time correlation functions in simple classical fluids. Our results agree quite favorably with the data generated by the computer experiments of Rahman and, in fact, represent a considerable improvement over existing kinetic-equation descriptions of time correlation functions in liquids.

It is proper at this point to compare such kinetic descriptions of time correlation functions with alternative descriptions based upon a generalization of the conventional hydrodynamic equations. ${ }^{5,6}$ Of 
course, the central idea behind all of these descriptions is to formally obtain exact expressions for time correlation functions in forms involving a "damping" or "memory" term which can then be easily modeled or approximated. The hydrodynamic approach develops exact expressions for the correlations among dynamical variables which have macroscopic counterparts in configuration space, e.g., mass or momentum densities. Such an approach possesses certain advantages for the study of liquids, since it yields explicit expressions for various physical quantities of interest (e.g., viscosity or conductivity) in terms of time correlation functions. It seems particularly well suited for the description of correlation functions in the range of small $k$ and $\omega$ dependence. To extend the modeled hydrodynamics description to higher $k$ and $\omega$ usually requires considerably more elaborate modeling (either by utilizing higher-order sum rules in the Martin-Yip ${ }^{6}$ formalism, or by considering a higher-dimensional description in the Akcasu-Daniels ${ }^{5}$ work).

In this regard, the kinetic-equation description is more suited for the description of the range of large $k$ and $\omega$. We have seen that it not only yields the proper large $k$ and $\omega$ limit of ideal gas behavior, but can be used as well to describe low $k$ and $\omega$ in the hydrodynamic regime, provided we adopt a suitable model of the damping kernel. In this sense, the modeled kinetic description provides a consistent means by which we can extend the hydrodynamic description to larger $k$ and $\omega$ [by calculating higher moments of $X(p, s)$ or $Y(p, s)]$. Unfortunately, the kinetic approach suffers from the complexities of modeling and obtaining a solution to the kinetic equation itself. It is, of course, much easier to model a function of $k$, as in the hydrodynamic description, than an operator, as we must in the kinetic approach. Moreover, there are very few "modeled collision operators" which will permit us to extract sufficient information about the behavior of $\mathrm{S}_{k}\left(\overrightarrow{\mathrm{p}}, \overrightarrow{\mathrm{p}}^{\prime \prime}\right.$, $t$ ) to allow a practical calculation of time correlation functions.

\section{ACKNOWLEDGMENTS}

This work has benefited rather substantially from numerous discussions with Professor Richard Osborn, Professor Noel Corngold, and Dr. Eugene Daniels. The authors are particularly grateful to Dr. Daniels for supplying the calculations of the elastic moduli $C_{i j}(k)$.

\section{APPENDIX A}

We wish to calculate the $t=0$ form of the damping kernel

$$
\begin{aligned}
& \varphi\left(\overrightarrow{\mathrm{p}}, \overrightarrow{\mathrm{p}}^{\prime}, 0\right)=\left[n M\left(\overrightarrow{\mathrm{p}}^{\prime}\right)\right]^{-1}\left\langle(1-P) \sigma^{*}\left(\overrightarrow{\mathrm{p}}^{\prime}\right)(1-P)_{\sigma}(\overrightarrow{\mathrm{p}})\right\rangle \\
& =\left[n M\left(\overrightarrow{\mathrm{p}}^{\prime}\right)\right]^{-1}\left\{\left\langle\sigma^{*}\left(\overrightarrow{\mathrm{p}}^{\prime}\right) \sigma(\overrightarrow{\mathrm{p}})\right\rangle\right. \\
& \left.-\mu(\overrightarrow{\mathrm{p}})\left\langle\sigma^{*}\left(\overrightarrow{\mathrm{p}}^{\prime}\right) \delta \rho_{k}\right\rangle\right\} \text {, } \\
& \text { where } \mu(\overrightarrow{\mathrm{p}}) \equiv \frac{-i \overrightarrow{\mathrm{k}} \circ \overrightarrow{\mathrm{p}}}{m} M(\overrightarrow{\mathrm{p}}) \frac{n g(k)}{1+n h(k)} \\
& \delta \rho_{k} \equiv \sum_{\alpha=1}^{N} e^{i \overrightarrow{\mathrm{k}} \cdot \overrightarrow{\mathrm{x}}^{\alpha}}-n \delta(\overrightarrow{\mathrm{k}})
\end{aligned}
$$

First calculate

$$
\left\langle\sigma^{*}\left(\overrightarrow{\mathrm{p}}^{\prime}\right) \delta \rho_{k}\right\rangle=\frac{-i \overrightarrow{\mathrm{k}} \cdot \overrightarrow{\mathrm{p}}^{\prime}}{m} n^{2} g(k) M\left(\overrightarrow{\mathrm{p}}^{\prime}\right) .
$$

Next calculate

$$
\begin{aligned}
& \left\langle\sigma^{*}\left(\overrightarrow{\mathrm{p}}^{\prime}\right) \sigma(\overrightarrow{\mathrm{p}})\right\rangle=\sum_{\alpha=1}^{N} \sum_{\beta=1}^{N}\left\langle e^{-i \overrightarrow{\mathrm{k}} \cdot \overrightarrow{\mathrm{x}}^{\alpha}} \overrightarrow{\mathrm{F}}^{\alpha} \cdot \frac{\partial}{\partial \overrightarrow{\mathrm{p}}^{\alpha}} \delta\left(\overrightarrow{\mathrm{p}}^{\prime}-\overrightarrow{\mathrm{p}}^{\alpha}\right) e^{i \overrightarrow{\mathrm{k}} \cdot \overrightarrow{\mathrm{x}}^{\beta}} \overrightarrow{\mathrm{F}}^{\beta} \cdot \frac{\partial}{\partial \overrightarrow{\mathrm{p}}^{\beta}} \delta\left(\overrightarrow{\mathrm{p}}-\overrightarrow{\mathrm{p}}^{\beta}\right)\right\rangle
\end{aligned}
$$

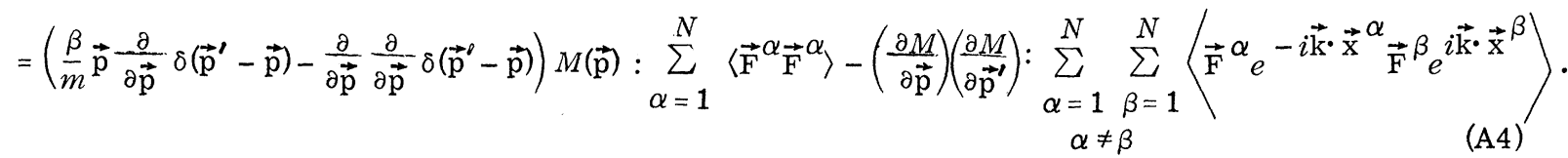

But if we note

$$
\begin{aligned}
& \sum_{\alpha}\left\langle F_{i}{ }^{\alpha} F_{j}{ }^{\alpha}\right\rangle=n \delta_{i j} D(0), \\
& \sum_{\alpha, \beta}^{\circ}\left\langle F_{i}{ }^{\alpha}{ }_{F}{ }_{j}{ }^{i} e^{i \overrightarrow{\mathrm{k}}^{\circ} \cdot \overrightarrow{\mathrm{x}}^{\alpha \beta}}\right\rangle=k_{i j}{ }_{j} \frac{n^{2} g(k)}{\beta^{2}}-n D_{i j}(k),
\end{aligned}
$$

then it is a straightforward calculation to combine (A3) and (A4), using (A5) and then integrating, to find

$$
\begin{aligned}
\int d \overrightarrow{\mathrm{p}}^{\prime} & \varphi\left(\overrightarrow{\mathrm{p}}, \overrightarrow{\mathrm{p}}^{\prime}, 0\right) \mathcal{S}_{k}\left(\overrightarrow{\mathrm{p}}^{\prime}, \overrightarrow{\mathrm{p}}^{\prime \prime}, t\right) \\
= & D(0)\left\{\frac{\partial}{\partial \overrightarrow{\mathrm{p}}} \cdot \frac{\partial}{\partial \overrightarrow{\mathrm{p}}}+\frac{\beta}{m} \frac{\partial}{\partial \overrightarrow{\mathrm{p}}} \cdot \overrightarrow{\mathrm{p}}\right\} \mathcal{G}_{k}\left(\overrightarrow{\mathrm{p}}, \overrightarrow{\mathrm{p}}^{\prime \prime}, t\right)
\end{aligned}
$$




$$
\begin{aligned}
& +\overrightarrow{\mathrm{p}} M(\overrightarrow{\mathrm{p}}) \cdot\left[\overrightarrow{\mathrm{k}} \overrightarrow{\mathrm{k}} n c(k) / m^{2}-\left(\beta^{2} / m^{2}\right) \overleftrightarrow{\mathrm{D}}(k)\right] \\
& +\int d \overrightarrow{\mathrm{p}}^{\prime} \overrightarrow{\mathrm{p}}^{\prime} G_{k}\left(\overrightarrow{\mathrm{p}}^{\prime}, \overrightarrow{\mathrm{p}}^{\prime \prime}, t\right) .
\end{aligned}
$$

\section{APPENDIX B}

We shall summarize the derivation of an exact expression for the transverse current-current correlation function as given by Akcasu and Daniels. ${ }^{5}$ Again utilizing the Mori-Zwanzig formalism, we choose the components of the state vector a as components of the mass current density and the stress tensor

$$
\begin{aligned}
a_{1}=J_{1}(k)= & \sum_{\alpha=1}^{N} p_{1}{ }^{\alpha} \exp \left(\overrightarrow{\mathrm{k}} \cdot \overrightarrow{\mathrm{x}}^{\alpha}\right), \\
a_{2}=\Pi_{31}(k)= & \sum_{\substack{\alpha=1 \\
N}}^{N}\left(p_{3} p_{1} / m\right) \exp \left(\overrightarrow{\mathrm{k}} \cdot \overrightarrow{\mathrm{x}}^{\alpha}\right) \\
& +\sum_{\substack{\alpha=1 \\
\alpha \neq \beta}}^{N} \sum_{\substack{\alpha \neq 1 \\
\alpha 1}}^{N} P_{3 \beta} \exp \left(i \overrightarrow{\mathrm{k}} \cdot \overrightarrow{\mathrm{x}}^{\alpha}\right),
\end{aligned}
$$

where

$$
P_{i j}^{\alpha \beta}=x_{i}^{\alpha \beta} \frac{\partial V^{\alpha \beta}}{\partial x_{j}^{\alpha}}\left(\frac{\exp \left(-i \overrightarrow{\mathrm{k}} \cdot \overrightarrow{\mathrm{x}}^{\alpha \beta}\right)-1}{i \overrightarrow{\mathrm{k}} \cdot \overrightarrow{\mathrm{x}}}\right)
$$

Then a straightforward calculation ${ }^{5}$ yields the frequency matrix

$$
\underline{\Omega}=k\left[\begin{array}{cc}
0 & 1 \\
C_{44}(k) / m n & 0
\end{array}\right] \text {. }
$$

Furthermore, since $(1-P) a_{1}=i k(1-P) a_{2}=0$, we find

$$
\varphi(t)=\left[\begin{array}{cc}
0 & 0 \\
0 & \varphi_{22}(t)
\end{array}\right]
$$

where $\varphi_{22}(t) \equiv\left\langle(1-P) \dot{a}_{2}{ }^{*}\right.$

$$
\left.\times \exp [i t(1-P) L](1-P) \dot{a}_{2}\right\rangle\left\langle a_{2} a_{2} *^{-1} .\right.
$$

Hence the generalized Langevin equation (1) for the set (B1) becomes

$$
\begin{gathered}
\frac{d a_{1}}{d t}-i k a_{2}(t)=0, \\
\frac{d a_{2}}{d t}-\frac{i k C_{44}(k)}{m n} a_{1}(t) \\
+\int_{0}^{t} d \tau \varphi_{22}(\tau) a_{2}(t-\tau)=f_{2}(t) .
\end{gathered}
$$

Now we see from (15) that

$$
g_{\perp}(k, t)=\left\langle a_{1}(t) a_{1}^{*}\right\rangle\left\langle a_{1} a_{1}^{*}\right\rangle^{-1}
$$

Hence, by multiplying the set (B6) by $a_{1}^{*}$, ensemble averaging, and then Laplace transforming in time, we can solve for

$$
\tilde{\partial}_{\perp}(k, s)=\left(s+\frac{k^{2} C_{44}(k) / m n}{s+\tilde{\varphi}_{22}(s)}\right)^{-1}
$$

or $\mathcal{J}_{\perp}(k, \omega)=\operatorname{Re}\left\{\left[i \omega+k^{2} \eta_{\perp}(k, \omega) / m n\right]^{-1}\right\}$,

where we define a $k$ - and $\omega$-dependent shear viscosity by

$$
\eta_{\perp}(k, \omega)=\frac{C_{44}(k)}{i \omega+\tilde{\varphi}_{22}(k, i \omega)} .
$$

As it stands, (B9) is still an exact expression for $\mathcal{J}_{\perp}(k, \omega)$. But the actual calculation of $\tilde{\varphi}_{22}(k, i \omega)$ is as difficult as that involved in calculating $J_{\perp}(k, \omega)$ directly; hence, Akcasu and Daniels chose to introduce a "Markovian" approximation by replacing $\tilde{\varphi}_{22}$ by its $\omega \rightarrow 0$ limit:

$$
\lim _{\omega \rightarrow 0} \tilde{\phi}_{22}(k, i \omega) \equiv \omega_{\perp}(k)
$$

for small frequencies $\omega$. Hence, $\mathscr{J}_{\perp}(k, \omega)$ becomes

$$
g_{\perp}(k, \omega)=\frac{\omega_{\perp}(k) k^{2} C_{44}(k) / m n}{\omega^{2} \omega_{\perp}^{2}(k)+\left[k^{2} C_{44}(k) / m n-\omega^{2}\right]^{2}} .
$$

However, even $\omega_{\perp}(k)$ as given by (B11) is extremely difficult to calculate. Hence, instead of calculating $\omega_{\perp}(k)$ directly, an attempt was made to model its $k$ dependence as follows: For small $k, \eta_{\perp}(k, 0)$, as defined in (B10), reduces to the usual shear viscosity $\eta_{S}$. Hence,

$$
\lim _{k \rightarrow 0} \omega_{\perp}(k)=C_{44}(O) / \eta_{s} .
$$

Furthermore, for large $k$ one expects to obtain the dispersion relation $\omega_{m}^{\perp}(k)$ characteristic of an ideal gas $^{16}$ (here, $\omega_{m}{ }^{\perp}(k)$ is that frequency at which $\mathcal{J}_{\perp}(k, \omega)$ assumes a maximum value for a given $k$ ). Noting that for an ideal gas

$$
J_{\perp}(k, \omega)=\left(\frac{\pi \beta m}{2 k^{2}}\right)^{1 / 2} \exp \left[-\frac{\omega^{2}}{k^{2}}\left(\frac{m \beta}{2}\right)\right],
$$

and hence $\omega_{m} \perp(k)=0$ for all $k$, Akcasu and Daniels required $\omega_{m} m^{\perp}(k)$, as calculated from (B12), to approach zero as $k \rightarrow \infty$. This leads to the requirement 


$$
\omega_{\perp}^{2}(k)-2 k^{2} C_{44}(k) / m n, \quad \text { as } k \rightarrow \infty .
$$

For intermediate $k$, a simple interpolation formula was used:

$$
\omega_{\perp}^{2}(k)=\frac{2 k^{2} C_{44}(k)}{m n}
$$

$$
+\frac{\omega_{\perp}{ }^{2}(0)-2 k^{2}\left[C_{44}(k)-n / \beta\right] / m n}{1+k^{2} / k_{0}^{2}}
$$

where the interpolation parameter was taken as $k_{0}=1.5 \AA^{-1}$
*Work supported in part by the National Science Foundation.

${ }^{1}$ A. Z. Akcasu and J. J. Duderstadt, Phys. Rev. 188, 479 (1969).

${ }^{2} \mathrm{R}$. Zwanzig, in Lectures in Theoretical Physics, edited by W. E. Brittin (John Wiley \& Sons, New York, 1961).

${ }^{3}$ H. Mori, Progr. Theoret. Phys. (Kyoto) 33, 423 (1965) .

${ }^{4}$ A. Rahman, in Neutron Inelastic Scattering (International Atomic Energy Agency, Vienna, 1968), Vol. I, p. 561.

${ }^{5}$ A. Z. Akcasu and E. Daniels, Phys. Rev. (to be published).

${ }^{6}$ C. H. Chung and S. Yip, Phys. Rev. 182, 323 (1969).

${ }^{7}$ B. Berne, J. Boon, and S. A. Rice, J. Chem. Phys. 45, 1086 (1966).

${ }^{8}$ M. Nelkin and S. Ranganathan, Phys. Rev. 164, 222 (1967).

${ }^{9}$ M. Nelkin and P. J. Ortoleva, in Neutron Inelastic Scattering (International Atomic Energy Agency, Vienna,
1968) Vol. I, p. 535; Phys. Rev. 181, 429 (1969).

${ }^{10}$ L. P. Kadanoff and P. C. Martin, Ann. Phys. (N. Y.) 24, 419 (1963).

${ }^{11}$ R. Zwanzig, Phys. Rev. 144, 170 (1966).

${ }^{12}$ J. Chihara, Progr. Theoret. Phys. (Kyoto) $\underline{41}, 285$ (1969).

${ }^{13}$ H. Mori, Progr. Theoret. Phys. (Kyoto) 34, 399 (1965).

${ }^{14} \mathrm{~A}$ closely related, although somewhat more general, approximation has been employed recently [J. L.

Lebowitz, J. K. Percus, and J. Sykes, Phys. Rev. 188, 487 (1969) ], to study time correlation functions using kinetic equations. These authors hence obtain and solve exactly a kinetic equation essentially identical to Eq. (12) of this work.

${ }^{15}$ P. Schofield, Proc. Phys. Soc. (London) 88, 149 (1966).

${ }^{16}$ This point is more apparent in the case of longitudinal current-current correlations, ${ }^{5}$ since $k$ may then be interpreted as the momentum transfer involved in a scattering (e.g., neutron) experiment.

\title{
Osmotic Pressure of Dilute Solutions of $\mathrm{He}^{3}$ in $\mathrm{He}^{4 \dagger}$
}

\author{
M. F. Wilson* and J. T. Tough \\ Department of Physics, Ohio State University, Columbus, Ohio 43210 \\ (Received 8 September 1969)
}

\begin{abstract}
The osmotic pressure of dilute solutions of $\mathrm{He}^{3}$ in liquid $\mathrm{He}^{4}$ has been measured at $0.32,0.65$, and $1.16^{\circ} \mathrm{K}$ for concentrations up to $2 \%$. "The technique does not require values of the $\mathrm{He}^{4}$ fountain pressure and is substantially more precise than previous methods. The results are analyzed in terms of the effective-interaction model of Bardeen, Baym, and Pines as extended to nonzero temperatures by Ebner. The results do not agree well with the theory.
\end{abstract}

\section{INTRODUCTION}

Dilute solutions of $\mathrm{He}^{3}$ in $\mathrm{He}^{4}$ have been the subject of a great deal of experimental and theoretical effort in the past few years. ${ }^{1}$ Bardeen, Baym, and Pines $(\mathrm{BBP})^{2}$ have successfully extended the LandauPomeranchuk ${ }^{3}$ model of solutions to finite concentrations and low temperatures by means of an effective $\mathrm{He}^{3}$ quasiparticle interaction $V_{k}$. Baym and Ebner ${ }^{4}$ have calculated a $V_{k}$ to fit the trans- port properties (spin diffusion ${ }^{5}$ and thermal conductivities $\left.^{6}\right)$ at low temperatures $\left(T \ll T_{F}\right.$, where $T_{F}$ is the Fermi temperature of the $\mathrm{He}^{3}$ component of the solution). This $V_{k}$ is in excellent agreement with that calculated from recently measured low-temperature static properties (heat of mixing, ${ }^{7}$ phase separation curve ${ }^{8}$ and osmotic pressure ${ }^{9}$ ). Ebner has recently calculated a new $V_{k}$ based upon exact solutions to the transport equations. ${ }^{10}$ This $V_{k}$ gives only fair agreement with the low tempera- 